Supporting Information for:

\title{
Elucidating Interfacial Stability between Lithium Metal Anode and LiPON via In situ Electron Microscopy
}

\section{Zachary D. Hood, ${ }^{1, \dagger}, \&$ Xi Chen, ${ }^{2, \dagger}$ Robert L. Sacci, ${ }^{3, \dagger}$ Xiaoming Liu, ${ }^{1}$ Gabriel M. Veith, ${ }^{3}$ Yifei Mo, ${ }^{4}$ Junjie Niu, ${ }^{2}$ Nancy J. Dudney, $3,{ }^{*}$ Miaofang Chi ${ }^{1, *}$}

${ }^{1}$ Center for Nanophase Materials Sciences, Oak Ridge National Laboratory, Oak Ridge, Tennessee 37831, USA

${ }^{3}$ Department of Materials Science and Engineering, CEAS, University of Wisconsin-Milwaukee, Milwaukee, Wisconsin 53211, USA

${ }^{3}$ Chemical Sciences Division, Oak Ridge National Laboratory, Oak Ridge, Tennessee 37831, USA

${ }^{4}$ Department of Materials Science and Engineering, University of Maryland, College Park, Maryland 20742, USA

${ }^{+}$These authors contributed equally to this publication.

*Correspondence to: Miaofang Chi (chim@ornl.gov), Nancy J. Dudney (dudneynj@ornl.gov), Junjie Niu (niu@uwm.edu) 


\section{Experimental Details:}

\section{Fabrication of half copper grids}

Copper grids (3 mm, 200 mesh, SPI supplies) were carefully mounted onto a polishing stage with epoxy melted at approximately $150{ }^{\circ} \mathrm{C}$. Next, the copper grid was polished with a Techprep ${ }^{\mathrm{TM}}$ polisher rotating at $140 \mathrm{rpm}$, which was fitted with a $3 \mu \mathrm{m} 8$ " Disk from Allied High Tech Products, Inc. The copper grid was polished in increments of approximately $0.005 \mathrm{~mm}$. Next, copper grids were removed from the polishing stage using acetone, sonicated for 30 minutes in acetone, and finally washed two times in ethanol and two times in methanol. The copper grid was then carefully mounted onto the sample stage for Low Angle Ion Milling, which was performed on a Low Angle

Ion Milling and Polishing System (Fischione Instruments). The ion milling was performed at 6 $\mathrm{kV}, 5 \mathrm{~mA}$ and $\sim 0.5 \mathrm{sccm}$ for at least 15 minutes at a milling angle of $15^{\circ}$ and an operating pressure of less than $4.0 \times 10^{-4}$ Torr. At this point, the copper grid is ready for depositing LiPON via reactive radio frequency magnetron sputtering.

\section{Fabrication of radio frequency sputtered LiPON on copper half grids}

LiPON solid electrolyte was deposited onto copper half grids by radio frequency (RF) magnetron sputtering following previously reported procedures. ${ }^{1-2}$ In short, thin LiPON films with thicknesses

$\sim 100 \mathrm{~nm}$ were sputtered from a home-made $\mathrm{Li}_{3} \mathrm{PO}_{4}$ target in the presences of a reactive $\mathrm{N}_{2}$ atmosphere (99.9995\%). These thin solid electrolyte films are amorphous as confirmed by electron diffraction patterns from TEM imaging. 


\section{Setup of the Nanofactory Holder and in situ biasing of the LiPON-Li interface}

The half copper grid was placed in the Nanofactory holder, as shown in Figure 1. Fresh Li metal anode (Alfa Aesar, 99.999\%) was then deposited on a tungsten wire by simply scratching the surface of the Li metal. The tungsten wire was connected to the piezo-controlled STM tip. The holder was immediately transferred to the scanning transmission electron microscope to avoid Li oxidation. Once in the TEM, in order to ensure that Li metal has minimal contaminants (e.g. LiOH, $\mathrm{Li}_{2} \mathrm{O}$, etc.), the surface layers of the Li metal anode were sculpted with an electron beam of a high dose rate of $\sim 800 \mathrm{pA}$ at several tilt angles. The absence of oxides on Li tip was then confirmed by EELS. By carefully positioning the Li metal covered tungsten tip with the piezo-electronic controller, the LiPON-Li interface was fabricated inside the microscope. In some cases, the electron beam was blocked immediately after touching LiPON with Li metal to form the interface. Using the unique setup, we could also bias the interface between 0 and $5 \mathrm{~V}$.

\section{S/TEM imaging and EELS spectroscopy}

Transmission electron microscopy (TEM) images were collected on an aberration-corrected FEI Titan operated at $300 \mathrm{kV}$ with a spatial resolution of $\sim 0.63$ Angstrom. EELS spectra were collected with a Gatan Quantum-865 EFTEM/EELS spectrometer. The EELS spectra were acquired at a dispersion of $0.1 \mathrm{eV}$ per channel with a convergence angle of $28 \mathrm{mrad}$ and a collection angle of $\sim 27 \mathrm{mrad}$. The acquisition time of EELS was 1s, during which the electron beam was scanned over a rectangular area perpendicular to the interface of $2 \times 6 \mathrm{~nm}$ area. As a result, the measurement indicates the spatially averaged interface signal of $2 \mathrm{~nm}$ thickness. the accumulative electron dose used for EELS analysis is $\sim 5 \times 10^{4} \mathrm{e} / \mathrm{nm}^{2}$, which was estimated by multiplying the acquisition time by the dose rate. The dose rate was measured using the microscope's fluorescent screen that had 
been previously calibrated using a pico-ammeter. The spectra images shown in Figure 5 was acquired under the same beam condition as that for EELS spectra, but with an acquisition time of $0.8 \mathrm{~s} /$ pixel with a pixel size of $\sim 1.3 \mathrm{~nm}$.

X-ray Photoelectron Spectroscopy Analysis

Samples for XPS were prepared by sputtering LiPON on a glass surface $(200 \mathrm{~nm})$ alongside the $\mathrm{Cu}$ grid substrate used in the TEM imaging experiments. The glass substrates were moved into an Ar-filled glovebox. One sample was touched by freshly cleaned Li metal foil for 10 seconds. The two samples were then transferred under vacuum to the XPS system. Measurements were collected using a PHI 3056 spectrometer with an MG anode source operating at $15 \mathrm{kV}$ and $23.3 \mathrm{~A}$. The elemental data was collected at a pass-energy of $11 \mathrm{eV}$ with $0.05 \mathrm{eV}$ steps and 100 iterations.

\section{Volume/Area Expansion Estimation}

Based on the synthesis methods, the stoichiometry of LiPON is $\mathrm{Li}_{2.9} \mathrm{PO}_{3.3} \mathrm{~N}_{0.46}$, according to previous reports. ${ }^{[1,2]}$ Table $\mathrm{S} 1$ displays the volume of $\mathrm{Li}_{3} \mathrm{P}, \mathrm{Li}_{3} \mathrm{~N}$ and $\mathrm{Li}_{2} \mathrm{O}$ as reaction products. There is about a $201.3 \%$ volume change based on the density and molar mass of the LiPON and reaction products. If we simplify the LiPON-Li interface topography to a semi-cylindrical model, taking the half-arc surface in semi-cylindrical model as the main observation direction, the estimated area expansion ratio is 1.43 , coinciding with the observed areal expansion ratio $(\sim 1.5)$.

Supplementary Video S1 In situ TEM observation of LiPON upon contacting with Li metal at a $5 \times$ playback speed.

Supplementary Video S2 In situ TEM observation of LiPON upon contacting with Li metal at a $1 \times$ playback speed and blanking the beam for $\sim 20$ seconds. 

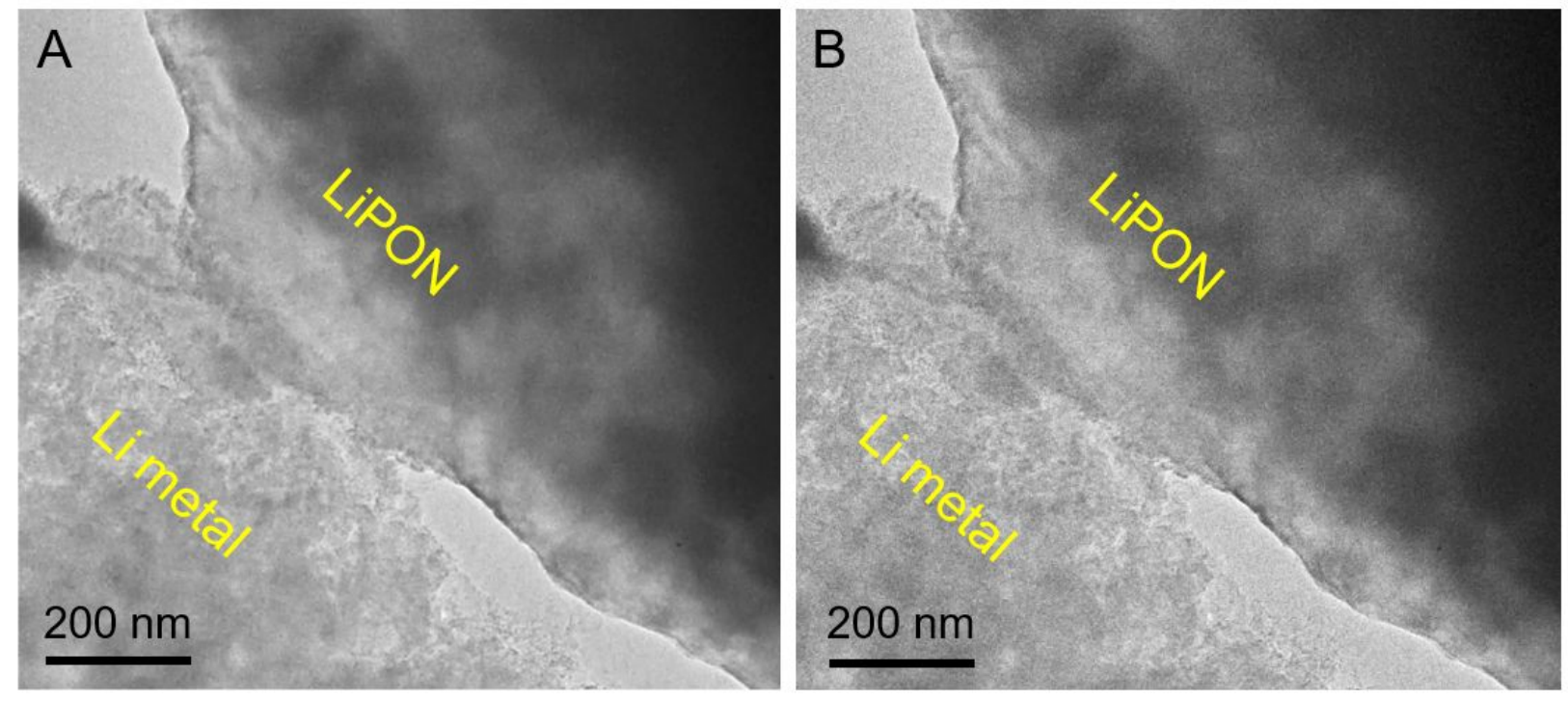

Figure S1. Evolution of the LiPON-Li interface A) before and B) after an external bias of 5V vs $\mathrm{Li} / \mathrm{Li}^{+}$. No observable changes were detected in TEM imaging after the external bias of $5 \mathrm{~V}$.
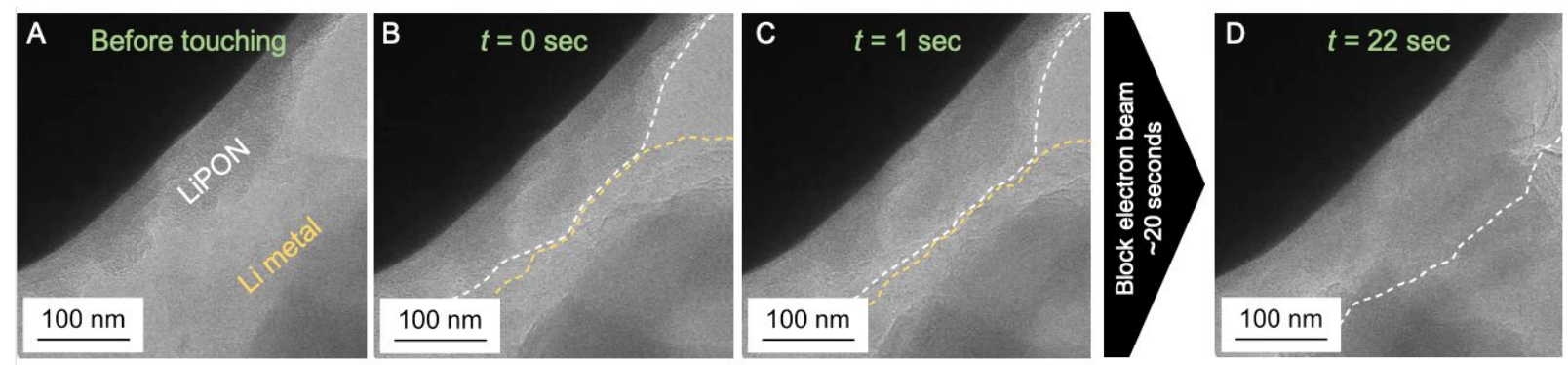

Figure S2. In situ TEM observation of the initial interphase formation upon first contacting Li metal with LiPON and immediately blocking the electron beam from time $t=1.5 \mathrm{~s}$ to $t=21.5 \mathrm{~s}$. probed by in situ TEM. The sequential TEM images show A) LiPON and Li metal before touching, B) LiPON and Li metal first touching $(e . g . t=0 \mathrm{~s}), \mathrm{C})$ the interphase formation at time $t=1 \mathrm{~s}$, and D) the interphase formation after blocking the electron beam for $20 \mathrm{~s}$. The LiPON and Li boundaries are marked by the white and yellow dashed lines, respectively, in (B) and (C). The white dashed line in (D) marked the boundary between the interphase and the Li metal; the interphase thickness was determined to be $c a .60 \mathrm{~nm}$. 


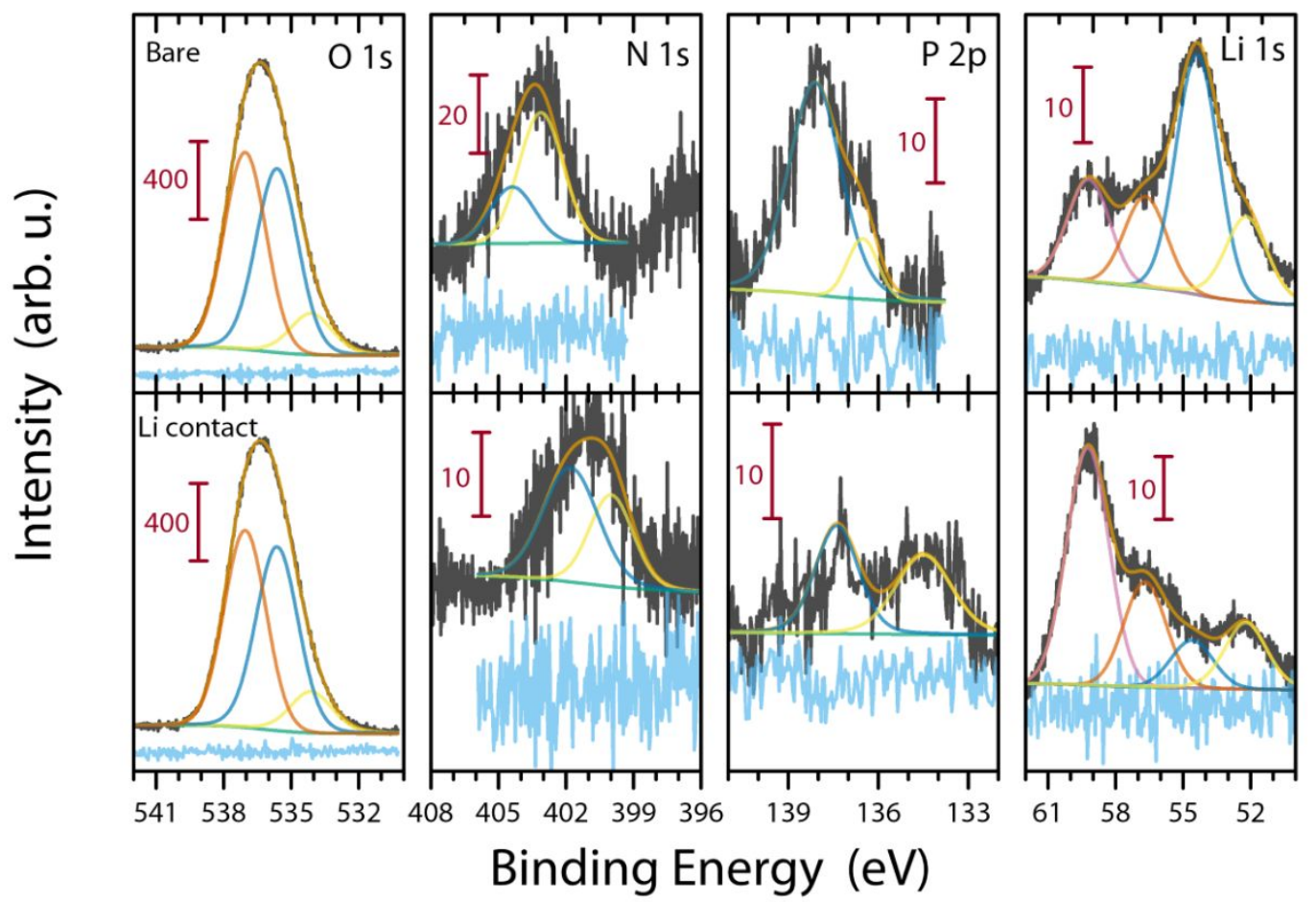

Figure S3. X-ray photoelectron spectroscopy (XPS) analysis of LiPON before (top) and after (bottom) touching Li metal for 10 seconds. The relevant atomic signals of $\mathrm{N}$ and $\mathrm{P}$ shift to lower electron binding energies upon Li contact, suggesting that they are become more reduced. The overall intensity of the P signals is substantially lowered due to Li contact, suggesting that there is less P present within the depth of interfacial region probed by XPS. This result agrees with the TEM finding results showing an oxide-rich interface followed by a P-rich sub-interface. The Li signal is greater with more high binding energy peak, further agreeing with the presence of more $\mathrm{Li}_{2} \mathrm{O}$ on the surface. 


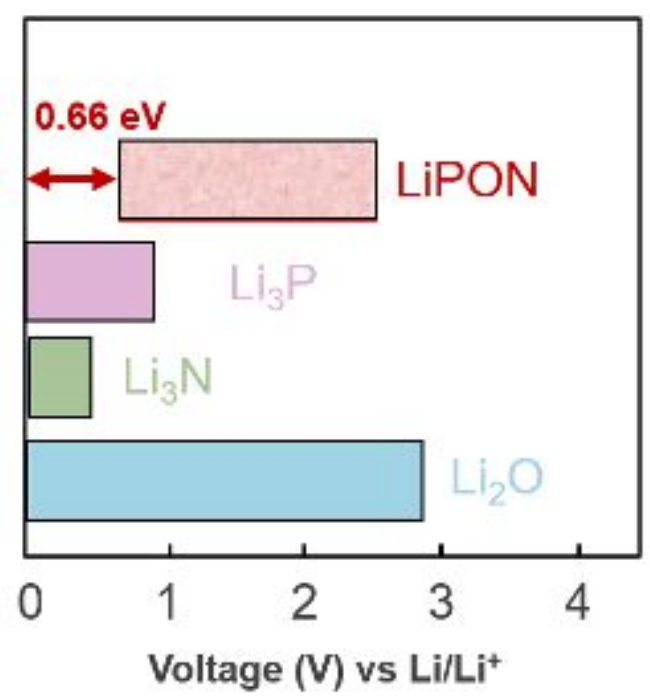

Figure S4. Calculated stability windows of $\mathrm{LiPON}, \mathrm{Li}_{3} \mathrm{P}, \mathrm{Li}_{3} \mathrm{~N}$, and $\mathrm{LiO}_{2}$ (replotted from Ref. [3]).

Table S1. Volume of $\mathrm{Li}_{2.99} \mathrm{PO}_{3.38} \mathrm{~N}_{0.41}, \mathrm{Li}_{3} \mathrm{P}, \mathrm{Li}_{3} \mathrm{~N}$, and $\mathrm{Li}_{2} \mathrm{O}$ based on standard molar masses and densities. If $\mathrm{LiPON}$ were to decompose to just $\mathrm{Li}_{3} \mathrm{P}, \mathrm{Li}_{3} \mathrm{~N}$, and $\mathrm{Li}_{2} \mathrm{O}$, each mole of LiPON would form 1 mole of $\mathrm{Li}_{3} \mathrm{P}, 0.4$ moles of $\mathrm{Li}_{3} \mathrm{~N}$, and 3.4 moles of $\mathrm{Li}_{2} \mathrm{O}$, together with a volume fraction of $\sim 53$ vol. $\% \mathrm{Li}_{2} \mathrm{O}$ (a poor ion conductor) and $\sim 77$ vol. $\%$ of both $\mathrm{Li}_{3} \mathrm{P}$ and $\mathrm{Li}_{3} \mathrm{~N}$ (better ion conductors). In principle, this interphase would be sufficiently $\mathrm{Li}^{+}$conducting and electronically insulating. Thus, interphases bearing similar chemistries and structures deserve particular attention in future optimization of high-performance SE/Li interfaces.

\begin{tabular}{|c|c|c|c|c|}
\hline Compound & $\begin{array}{c}\text { Molar mass } \\
(\mathbf{g} / \mathbf{m o l})\end{array}$ & Density $\left(\mathbf{g} / \mathbf{c m}^{\mathbf{3}}\right)$ & Mole & Volume (cm \\
\hline $\mathrm{Li}_{2.99} \mathrm{PO}_{3.38} \mathrm{~N}_{0.41}$ & 111.55 & 2.3 & 1.0 & 48.49 \\
\hline $\mathrm{Li}_{3} \mathrm{P}$ & 51.796 & 1.44 & 1.0 & 35.97 \\
\hline $\mathrm{Li}_{3} \mathrm{~N}$ & 34.823 & 1.27 & 0.46 & 12.61 \\
\hline $\mathrm{Li}_{2} \mathrm{O}$ & 29.882 & 2.01 & 3.3 & 49.06 \\
\hline
\end{tabular}




\section{References:}

1) Lacivita, V.; Westover, A. S.; Kercher, A.; Phillip, N. D.; Yang, G.; Veith, G.; Ceder, G.; Dudney, N. J. Journal of the American Chemical Society 2018, 140, 11029.

2) Li, J.; Ma, C.; Chi, M.; Liang, C.; Dudney, N. J. Advanced Energy Materials 2015, 5, 1401408.

3) Zhu, Y.; He, X.; Mo, Y. Origin of Outstanding Stability in the Lithium Solid Electrolyte Materials: Insights from Thermodynamic Analyses Based on First-Principles Calculations. ACS Appl. Mater. Interfaces 2015, 7 (42), 23685-23693. 